\title{
Robustness of a relaxation oscillator
}

\author{
Tryphon T. Georgiou ${ }^{1}$ and Malcolm C. Smith ${ }^{2}$
}

\begin{abstract}
For a relaxation oscillator which consists of a relay-hysteresis in feedback with negative integral action we prove that the oscillatory behaviour is robust to perturbations in the dynamical component of the feedback loop which are sufficiently small in a gap sense.
\end{abstract}

Keywords: Nonlinear systems, limit cycles, dynamic uncertainty, gap metric.

\section{DEDICATION}

George Zames was a mentor and a friend. In our hearts and minds he will always be remembered with warmth and admiration for his generous personality and leadership, and his many contributions to the foundations of modern robust control. To his memory we dedicate this paper.

\section{INTRODUCTION}

A CENTRAL THEME in George Zames' investigations was to quantify the role of feedback in A combating modelling uncertainty. To this end, in his joint work with Ahmed K. El-Sakkary [34], he set the goal of seeking in full generality a "description of the tolerable uncertainties." This work gave rise to a suitable metric topology having the desired property that feedback stability is maintained in a small neighbourhood of a nominally stable feedback system. The metric used is known as the gap metric.

To date, this paradigm has been studied for both linear and nonlinear systems in the neighbourhood of a fixed operating condition (see [5-11, 14,23,26,27] and the references therein). In the present work we seek to extend this paradigm to a new situation-a nonlinear feedback oscillator. Such systems are not globally stable in an input-output sense. Moreover, the closeness between responses of such systems is not conveniently assessed with the usual norm-based measures. Nevertheless, with appropriate modifications, we are able to show that the basic ideas of the paradigm generalize to this new context.

Nonlinear oscillations are encountered in a large variety of physical phenomena from chemical reactions and interacting populations [21, pp. 154, 180], to circadian processes [29, pp. 169, 173], to neurosciences [3, p. 41], and to the dynamics of Cepheid variables in Astrophysics [18, p. 106]. Although the phenomenon of limit cycle oscillation appears ubiquitous, and therefore it must be fairly robust, there is little known about robustness of the respective mathematical models. In fact, the extensive mathematical literature on relaxation oscillators focuses on conditions for limit cycles to exist in a given system, on entrainment by external signals, and on the effects of parametric or stateequation uncertainty in fixed-order models (see [1,2,12,13,15,16,19,20,25] and the references therein). In contrast the gap approach to uncertainty allows for changes in the dynamic order of the system including the possibility of infinite-dimensional elements such as time-delays.

In our analysis we focus on a common type of oscillator, sometimes called a relaxation oscillator, where negative integral action drives a bistable system via a feedback interconnection. The bistable element in the feedback path is in general a dynamic hysteresis-type of nonlinearity. When the element is fixed in either of its two states, there is a build-up by the integral action in a direction which forces it into the other state, and so on. The most widely referenced example in the engineering literature is

\footnotetext{
${ }^{1}$ Department of Electrical and Computer Engineering, University of Minnesota, MN 55455, U.S.A.

${ }^{2}$ Department of Engineering, University of Cambridge, Cambridge, CB2 1PZ, U.K.

This research was supported in part by the NSF, AFOSR, and EPSRC.
} 
the van der Pol oscillator (see [15, p. 288], [12]). This paper considers the simple relaxation oscillator of Figure 1 where the hysteresis is an ideal relay with infinitely fast transition between the two states, while the dynamical component is a simple integrator. However, the authors believe that the approach

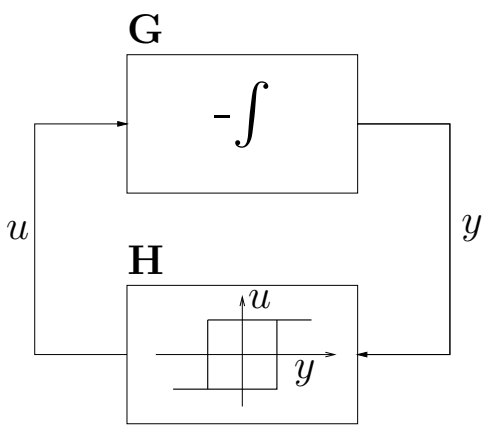

Fig. 1. Relay-relaxation oscillator

of the paper is amenable to generalization to other nonlinear feedback oscillators.

The paper is structured as follows. Section II describes the mathematical framework for analyzing the relaxation oscillator and establishes well-posedness. Section III discusses a notion of distance between oscillatory signals. Section IV presents some background theory and derives the main result of the paper (Theorem 1) which gives a bound on the amount of modelling uncertainty, measured in the gap metric, which guarantees that oscillatory behaviour persists for the uncertain system. Section $\mathrm{V}$ considers a specific class of perturbations of the negative integrator in the relay oscillator and gives robustness bounds by direct calculations and by application of Theorem 1 .

\section{FEEDBACK SYSTEMS WITH RELAY-HYSTERESIS}

A relay-hysteresis $\mathbf{H}(\cdot)$ is defined for a continuous input $y(t)$ for which $y(0)=0$ (see e.g., [24, p. $66])$. The output $u(t)$ takes values from the set $\{-1,+1\}$ and can be determined from:

(i) $u(0)=1$.

(ii) $u(t)$ is +1 when $y(t) \geq+1$ and -1 when $y(t) \leq-1$.

(iii) Suppose $y\left(t_{0}\right)>-1$ and $u\left(t_{0}\right)=+1$ for some $t_{0} \geq 0$. Then $u(t)=+1$ on any interval $\left[t_{0}, t_{1}\right)$ for which $y(t)>-1$.

(iv) Suppose $y\left(t_{0}\right)<+1$ and $u\left(t_{0}\right)=-1$ for some $t_{0} \geq 0$. Then $u(t)=-1$ on any interval $\left[t_{0}, t_{1}\right)$ for which $y(t)<+1$.

Condition (i) is due to the inherent "memory" of the system which requires that the "state" $u(\cdot)$ is specified at the initial time $t=0$.

The analysis of feedback systems with relay elements requires care due to the discontinuous nature of the outputs of such elements. To establish well-posedness of the nominal and perturbed feedback loops, i.e. existence and uniqueness of solution in the presence of a suitable class of external disturbance signals, the classical approach of using Banach's contraction mapping theorem [4,28,30-33] is not applicable. On the other hand, providing arbitrarily fast switching can be avoided, existence and uniqueness of solution can follow in a straightforward way by integrating the dynamic element of the feedback loop over successive intervals where the output of the relay-hysteresis element is constant.

If it is known a priori that the input to the hysteresis element is continuous on $[0, \infty)$ then there can be no finite limit point of switching times. (This can be seen by assuming to the contrary and observing a contradiction to the input being continuous at the limit point, or, by applying Proposition 3 with $\mathbf{G}=0$.) However, this approach is not available when the input is constructed successively over intervals which may become arbitrarily small. To deal with this problem we will consider dynamical elements of the nominal and perturbed feedback loops whose outputs have a Lipschitz property, and thereby establish a lower bound on the time between switches of the relay. 


\section{A. Choice of Signal Spaces and Systems}

Let $\mathcal{L}_{\infty}[0, \infty)$ denote the Lebesgue space with the usual sup norm, and $C[0, \infty)$ its subspace of continuous functions. Let

$$
\begin{aligned}
\operatorname{Lip}[0, \infty)= & \{y(t), t \in[0, \infty): y(0)=0, \text { and satisfies a Lipschitz condition } \\
& \left.\mathrm{C}_{T}=\sup \left\{\frac{|y(s)-y(t)|}{|s-t|}: s \neq t, \text { and } s, t \in[0, T)\right\}<\infty, \text { for all } 0<T<\infty\right\} .
\end{aligned}
$$

The Lipschitz constant $\mathrm{C}_{T}$ may depend on $y$ and the length $T$ of the interval, but is finite. We now define the following input and output spaces:

$$
\begin{aligned}
\mathcal{U} & =\mathcal{L}_{\infty}[0, \infty) \\
\mathcal{Y} & =\{y(t) \in C[0, \infty): y(0)=0\}
\end{aligned}
$$

The choice of $\mathcal{U}$ and $\mathcal{Y}$ is dictated by the fact that the output of the relay-hysteresis is discontinuous, while the input is required to be continuous.

We consider linear dynamical systems defined by an integral operator

$$
\mathbf{G}: u(t) \mapsto y(t)=\int_{0}^{t} g(t-\tau) u(\tau) d \tau,
$$

where $u(t) \in \mathcal{U}$ and the kernel $g(t)$ is piecewise Lipschitz, i.e., for any $T>0$ there are finitely many intervals $\left[0, \tau_{1}\right), \ldots,\left[\tau_{m}, T\right)$ such that $|g(s)-g(t)|<C|s-t|$ where $s, t$ belong to the same subinterval and $C$ is a constant which may depend on $T$. The class of such systems will be designated by $\mathbb{G}$. We believe that the theory presented in this paper extends to a much wider class which includes nonlinear time-varying systems as well.

Proposition 1: The range of $\mathbf{G} \in \mathbb{G}$ is a linear submanifold of $\operatorname{Lip}[0, \infty)$.

Proof: Let $u \in \mathcal{L}_{\infty}[0, \infty)$. The kernel $g$ is measurable, and so is the product $u(\tau) g(t-\tau)$ on any finite interval. Let $T>0$ and take $t, t+\delta$ in $[0, T)$. Then

$$
\begin{aligned}
|y(t+\delta)-y(t)| & =\left|\int_{0}^{t+\delta} u(\tau) g(t+\delta-\tau) d \tau-\int_{0}^{t} u(\tau) g(t-\tau) d \tau\right| \\
& =\left|\int_{0}^{t} u(\tau)(g(t+\delta-\tau)-g(t-\tau)) d \tau+\int_{t}^{t+\delta} u(\tau) g(t+\delta-\tau) d \tau\right| \\
& \leq\|u\|_{\infty}\left(\int_{0}^{t}|g(t+\delta-\tau)-g(t-\tau)| d \tau+\int_{t}^{t+\delta}|g(t+\delta-\tau)| d \tau\right) .
\end{aligned}
$$

Clearly

$$
\int_{t}^{t+\delta}|g(t+\delta-\tau)| d \tau \leq \delta \sup _{\tau \in[0, T]}|g(\tau)|
$$

To bound the first integral let $\left[0, \tau_{1}\right),\left[\tau_{1}, \tau_{2}\right), \ldots,\left[\tau_{m}, T\right)$ denote intervals where $g$ is Lipschitz continuous, and assume that $\mathrm{C}$ is an upper bound on the Lipschitz constants in all such subintervals. Then,

$$
\int_{0}^{t}|g(t+\delta-\tau)-g(t-\tau)| d \tau \leq \delta \mathrm{C} T+2 \delta m \cdot \sup _{\tau \in[0, T]}|g(\tau)| .
$$

This is due to the fact that $t \leq T$ and either $|g(t+\delta-\tau)-g(t-\tau)| \leq \mathrm{C} \delta$, or, in at most $m$ intervals of length $\delta,|g(t+\delta-\tau)-g(t-\tau)| \leq 2 \sup _{\tau \in[0, T]}|g(\tau)|$. We conclude that

$$
|y(t+\delta)-y(t)| \leq \delta \mathrm{C}_{1}
$$


where the constant $\mathrm{C}_{1}$ depends on $T$. That $y(0)=0$ follows by continuity of the integral since $g(t-\tau) u(\tau)$ is bounded near 0 .

\section{$B$. Well-posedness of relay feedback systems}

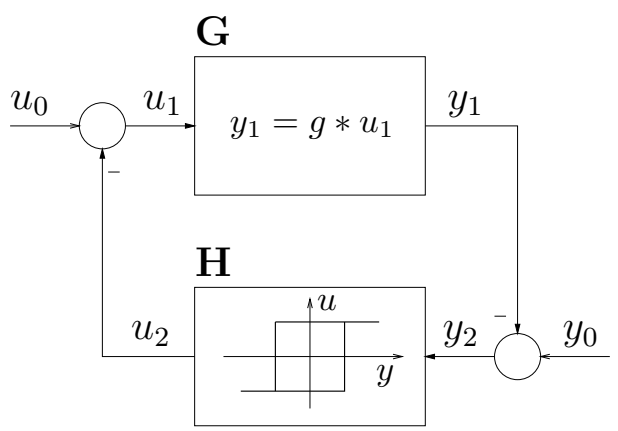

Fig. 2. Relay feedback system with external disturbances

We consider the relay feedback system of Figure 2 where external disturbances are added at each node. This feedback system will be denoted by $[\mathbf{G}, \mathbf{H}]$. We begin by considering the case of the nominal oscillator where $\mathbf{G}$ is a negative integrator. In this case

$$
y_{1}(t)=\int_{0}^{t}\left(\mathbf{H}\left(y_{0}-y_{1}\right)(\tau)-u_{0}(\tau)\right) d \tau,
$$

with $y_{0} \in \mathcal{Y}$, and $u_{0} \in \mathcal{U}$. The algebraic equations:

$$
\begin{aligned}
y_{2} & =y_{0}-y_{1}, \\
u_{2} & =\mathbf{H}\left(y_{0}-y_{1}\right), \\
u_{1} & =u_{0}-u_{2},
\end{aligned}
$$

determine the remaining variables $y_{2}, u_{2}, u_{1}$ in terms of $y_{1}$ and the external disturbances $y_{0}, u_{0}$.

Proposition 2: For any $u_{0} \in \mathcal{U}$ and $y_{0} \in \mathcal{Y},(1)$ has a unique solution $y_{1} \in \mathcal{Y}$. The remaining signals in the feedback loop satisfy: $u_{1}, u_{2} \in \mathcal{U}$ and $y_{2} \in \mathcal{Y}$.

Proof: We first establish existence of solution. Define $t_{1}>0$ to be the smallest $t$ for which $\rho_{1}(t):=y_{0}(t)-\int_{0}^{t}\left(1-u_{0}(\tau)\right) d \tau=-1$. If $\rho_{1}(t)>-1$ for all $t \geq 0$ we set $t_{1}=\infty$. In either case we observe that $\mathbf{H}\left(\rho_{1}\right)(t)=+1$ on $\left[0, t_{1}\right)$ and hence $y_{1}(t):=\int_{0}^{t}\left(1-u_{0}(\tau)\right) d \tau$ satisfies (1). This leads to a solution of the feedback equations by defining $y_{2}, u_{2}, u_{1}$ from (2), (3), and (4). If $t_{1}=\infty$, no further construction is required, so we assume that $t_{1}<\infty$. Now define $t_{2}>t_{1}$ to be the smallest $t$ for which $\rho_{2}(t):=y_{0}(t)-y_{1}\left(t_{1}\right)-\int_{t_{1}}^{t}\left(-1-u_{0}(\tau)\right) d \tau=1$. Since $\mathbf{H}\left(\rho_{2}\right)(t)=-1$ on $\left[t_{1}, t_{2}\right), y_{1}(t)=y_{1}\left(t_{1}\right)+\int_{t_{1}}^{t}\left(-1-u_{0}(\tau)\right) d \tau$ satisfies $(1)$, leading to a solution of the feedback equations on $\left[0, t_{2}\right)$. In a similar fashion we can extend the solution to an interval $\left[0, t_{k}\right)$ successively for $t_{k}>t_{k-1}$ etc. To complete the proof we need to show that $t_{k} \rightarrow \infty$ as $k \rightarrow \infty$. Assume to the contrary that $t_{f}:=\lim _{k \rightarrow \infty} t_{k}<\infty$. By definition of $y_{1}(t)$,

$$
\left|y_{1}\left(t_{k}\right)-y_{1}\left(t_{k-1}\right)\right| \leq\left(1+\left\|u_{0}\right\|_{\infty}\right)\left(t_{k}-t_{k-1}\right),
$$

for all $k$. Since $y_{0}(t)$ is continuous at $t_{f}$, then $\left|y_{0}\left(t_{k}\right)-y_{0}\left(t_{f}\right)\right| \leq 1 / 2$ for $k$ sufficiently large. Thus

$$
\begin{aligned}
2=\left|\rho_{k}\left(t_{k}\right)-\rho_{k-1}\left(t_{k-1}\right)\right| & =\left|y_{0}\left(t_{k}\right)-y_{1}\left(t_{k}\right)-y_{0}\left(t_{k-1}\right)+y_{1}\left(t_{k-1}\right)\right| \\
& \leq\left|y_{0}\left(t_{k}\right)-y_{0}\left(t_{k-1}\right)\right|+\left|y_{1}\left(t_{k}\right)-y_{1}\left(t_{k-1}\right)\right| \\
& \leq 1+\left(1+\left\|u_{0}\right\|_{\infty}\right)\left(t_{k}-t_{k-1}\right)
\end{aligned}
$$


for $k$ sufficiently large, which gives a contradiction.

We now turn to the question of uniqueness of solution. Assume that there exists a solution $y_{1} \in \mathcal{Y}$ of equation (1). By continuity of $y_{0}(t), y_{1}(t)$, there exists an $\epsilon>0$ so that $y_{0}(t)-y_{1}(t)>-1$ for all $t \in[0, \epsilon)$. Thus, (1) implies that $y_{1}(t)=\int_{0}^{t}\left(1-u_{0}(\tau)\right) d \tau$ on $[0, \epsilon)$, which is the same solution as obtained in the existence part. The solution continues to agree with that of the existence part on $\left[0, t_{1}\right)$, and then successively on subsequent intervals by similar reasoning.

For the general case where $\mathbf{G} \in \mathbb{G}$ the feedback equations become

$$
y_{1}(t)=\int_{0}^{t} g(t-\tau)\left(u_{0}(\tau)-\mathbf{H}\left(y_{0}-y_{1}\right)(\tau)\right) d \tau,
$$

together with (2),(3), and (4).

Proposition 3: For any $u_{0} \in \mathcal{U}$ and $y_{0} \in \mathcal{Y},(5)$ has a unique solution $y_{1} \in \mathcal{Y}$. The remaining signals in the feedback loop satisfy: $u_{1}, u_{2} \in \mathcal{U}$ and $y_{2} \in \mathcal{Y}$.

Proof: The proof of existence proceeds in a similar way to that of Proposition 2. Let $\rho_{1}(t):=$ $y_{0}(t)-\mathbf{G}\left(u_{0}(t)-1\right)$ and note that $\rho_{1}(t)$ is continuous with $\rho_{1}(0)=0$. Let $t_{1}>0$ be the smallest $t$ for which $\rho_{1}(t)=-1$, or set $t_{1}=\infty$ if $\rho_{1}(t)>-1$ for all $t \geq 0$. Then $\mathbf{H}\left(\rho_{1}\right)(t)=+1$ on $\left[0, t_{1}\right)$ and $y_{1}(t)=\mathbf{G}\left(u_{0}(t)-1\right)$ gives a solution of the feedback equations on $\left[0, t_{1}\right)$. As in Proposition 2 , the solution can be extended on successive intervals $\left[t_{k}, t_{k+1}\right)$. If $t_{f}:=\lim _{k \rightarrow \infty} t_{k}<\infty$ then, for all $k$,

$$
\left|y_{1}\left(t_{k}\right)-y_{1}\left(t_{k+1}\right)\right| \leq C_{t_{f}}\left(t_{k}-t_{k-1}\right)
$$

for some $C_{t_{f}}<\infty$, which may depend on $\left\|u_{0}\right\|_{\infty}$, see Proposition 1. A contradiction to the finiteness of $t_{f}$ follows as before. The proof of uniqueness of solution also follows as in Proposition 2.

\section{When ARE TWO OSCILlATORY SIGNALS ClOSE?}

A new feature in the problem of robustness of limit cycle oscillations is the difficulty of using the norm of the difference of two signals over the semi-infinite time-axis to quantify closeness. This is because oscillatory trajectories can get "out of step" in time due to perturbations. Two possible ways of dealing with this - restriction to compact time intervals and analysis in a phase space-have drawbacks. In the first case, allowable perturbations need to become smaller and smaller as the timeinterval is increased. In the second case, there may not be a common phase-space for the nominal and perturbed oscillator, e.g. when there is a difference in model order or when time-delays are introduced. Accordingly we introduce the device of allowing the time-axis to be re-scaled for one of the signals to be compared. Then, a notion of distance between oscillatory signals can be defined by combining the norm of their difference with the size of the chosen scaling. We now formalize such a distance measure.

Let $\mathcal{W}:=\mathcal{U} \times \mathcal{Y}$ and define:

$$
d\left(w_{1}(t), w_{2}(t)\right):=\inf \left\{\left\|w_{1}(t)-w_{2}(\sigma(t))\right\|_{\infty}+\sup _{t} \frac{|\sigma(t)-t|}{t}: \text { for } \sigma \in \mathcal{K}_{\infty}\right\},
$$

where $\mathcal{K}_{\infty}$ denotes the set of continuous monotonically non-decreasing functions $\sigma$ of $t \in[0, \infty]$ such that $\sigma(0)=0$ and $\sigma(\infty)=\infty$. For convenience, in the sequel, we use the notation $\sigma w(t):=w(\sigma(t))$. We note that a similar notion of distance has also been considered by S. Varigonda (personal communication).

\section{Robustness AnALYsis}

Our analysis relies on the formalism developed in [9-11]. In particular, we consider the relay oscillator in the standard feedback interconnection of Figure 2. We denote by $\mathcal{W}:=\mathcal{U} \times \mathcal{Y}$ the "ambient space" 
where input/output signals reside and consider the graphs of systems as subsets of $\mathcal{W}$, i.e.,

$$
\begin{gathered}
\mathcal{M}:=\operatorname{graph}(\mathbf{G}):=\left\{\left(\begin{array}{l}
u \\
y
\end{array}\right): y=\mathbf{G} u, u \in \mathcal{U}, y \in \mathcal{Y}\right\} \subset \mathcal{W}, \\
\mathcal{N}:=\operatorname{graph}(\mathbf{H}):=\left\{\left(\begin{array}{l}
u \\
y
\end{array}\right): u=\mathbf{H} y, y \in \mathcal{Y}, u \in \mathcal{U}\right\} \subset \mathcal{W} .
\end{gathered}
$$

The equations specifying the feedback interconnection can be written as $w_{0}=w_{1}+w_{2}$ where

$$
\begin{aligned}
w_{0} & :=\left(\begin{array}{l}
u_{0} \\
y_{0}
\end{array}\right) \in \mathcal{W}, \\
w_{1} & :=\left(\begin{array}{l}
u_{1} \\
y_{1}
\end{array}\right) \in \mathcal{M}, \\
w_{2} & :=\left(\begin{array}{l}
u_{2} \\
y_{2}
\end{array}\right) \in \mathcal{N} .
\end{aligned}
$$

The theory [10, Sections II and VIII] makes extensive use of the feedback map from external disturbances to the input and output of one of the two components of the feedback loop; e.g., in the case $\mathbf{G}$, of the map

$$
\Pi_{\mathcal{M} \| \mathcal{N}}: \mathcal{W} \rightarrow \mathcal{M}: w_{0} \mapsto w_{1}
$$

This is often referred to as a parallel projection operator - a terminology which reflects a geometric interpretation discussed in [5] and [10, Equation 1]. There is also a complementary parallel projection

$$
\Pi_{\mathcal{N} \| \mathcal{M}}: \mathcal{W} \rightarrow \mathcal{N}: w_{0} \mapsto w_{2}
$$

and moreover

$$
\Pi_{\mathcal{M} \| \mathcal{N}}+\Pi_{\mathcal{N} \| \mathcal{M}}=\mathbf{I}
$$

Next, the "distance" between dynamical systems is quantified by the distance to the identity of a suitable map which relates the input-output trajectories of the two systems. More precisely, if $\mathcal{M}_{1}$ denotes the graph of a perturbed system $\mathbf{G}_{1}$, we search over all causal maps $\boldsymbol{\Phi}_{\mathcal{M}}$ which map $\mathcal{M}$ bijectively onto $\mathcal{M}_{1}$ with $\boldsymbol{\Phi}_{\mathcal{M}} 0=0$ and select one which differs least from the identity. A nonlinear generalization of the gap metric can be based on the quantity $\inf _{\boldsymbol{\Phi}_{\mathcal{M}}}\left\{\left\|\left.\left(\mathbf{I}-\boldsymbol{\Phi}_{\mathcal{M}}\right)\right|_{\mathcal{M}}\right\|\right\}$ (see $[10]$ ).

We follow [10, Section VIII] and compare the response of two systems $\mathbf{G}, \mathbf{G}_{1}$ to a common external signal $w_{0}$. All we need in the current framework is a surjective map $\boldsymbol{\Phi}_{\mathcal{M}}$ from $\mathcal{M}$ onto $\mathcal{M}_{1}$. Let $w_{0}=m_{1}+n$ be the unique decomposition with $m_{1} \in \mathcal{M}_{1}$ and $n \in \mathcal{N}$, and note that

$$
m_{1}=\boldsymbol{\Pi}_{\mathcal{M}_{1} \| \mathcal{N}} w_{0} \text { and } n=\Pi_{\mathcal{N} \| \mathcal{M}_{1}} w_{0}
$$

The equation

$$
m_{1}=\boldsymbol{\Phi}_{\mathcal{M}} m
$$

must have a solution $m \in \mathcal{M}$ due to surjectivity of $\boldsymbol{\Phi}_{\mathcal{M}}$. Define $x_{0}=m+n$ and observe that $m=\Pi_{\mathcal{M} \| \mathcal{N}} x_{0}$ and $n=\Pi_{\mathcal{N} \| \mathcal{M}} x_{0}$, from which we obtain

$$
m_{1}=\Pi_{\mathcal{M}_{1} \| \mathcal{N}} w_{0}=\boldsymbol{\Phi}_{\mathcal{M}} \Pi_{\mathcal{M} \| \mathcal{N}} x_{0} .
$$

It follows that

$$
\begin{aligned}
w_{0} & =n+m_{1} \\
& =\boldsymbol{\Pi}_{\mathcal{N} \| \mathcal{M}} x_{0}+\boldsymbol{\Phi}_{\mathcal{M}} \boldsymbol{\Pi}_{\mathcal{M} \| \mathcal{N}} x_{0} \\
& =\left(\mathbf{I}+\left(\boldsymbol{\Phi}_{\mathcal{M}}-\mathbf{I}\right) \boldsymbol{\Pi}_{\mathcal{M} \| \mathcal{N}}\right) x_{0}
\end{aligned}
$$


The latter equation will be used to bound $x_{0}$. By subtracting $\sigma \boldsymbol{\Pi}_{\mathcal{M} \| \mathcal{N}} w_{0}$ from both sides of (6) and rearranging terms, we obtain

$$
\left(\sigma \boldsymbol{\Pi}_{\mathcal{M} \| \mathcal{N}}-\boldsymbol{\Pi}_{\mathcal{M}_{1} \| \mathcal{N}}\right) w_{0}=\left(\mathbf{I}-\boldsymbol{\Phi}_{\mathcal{M}}\right) \boldsymbol{\Pi}_{\mathcal{M} \| \mathcal{N}} x_{0}+\sigma \boldsymbol{\Pi}_{\mathcal{M} \| \mathcal{N}} w_{0}-\boldsymbol{\Pi}_{\mathcal{M} \| \mathcal{N}} x_{0}
$$

where $\sigma$ is any scaling of the time axis. This last equation will be our main tool for showing robustness of oscillations. The aim is to show that if the gap between the two dynamical components $\mathbf{G}$ and $\mathbf{G}_{1}$ is small then the left hand side of the equation can be made small by suitable choice of a ("small") $\sigma$, which amounts to closeness in the sense of Section III. Our main result is stated below.

Theorem 1: Let $\mathbf{G}$ be the negative integrator, $\mathbf{G}_{1}$ be an arbitrary element in $\mathbb{G}$, and $\mathbf{H}$ be the relayhysteresis defined in Section II, and denote their graphs by $\mathcal{M}, \mathcal{M}_{1}, \mathcal{N}$, respectively. If there exists a surjective map $\Phi_{\mathcal{M}}: \mathcal{M} \rightarrow \mathcal{M}_{1}$ such that

$$
\left\|\left.\left(\mathbf{I}-\mathbf{\Phi}_{\mathcal{M}}\right)\right|_{\mathcal{M}}\right\| \leq \epsilon<\frac{1}{3}
$$

then there exists a function $\sigma \in \mathcal{K}_{\infty}$ such that

$$
\sup _{t} \frac{|\sigma(t)-t|}{t} \leq \frac{4 \epsilon(1-\epsilon)}{(1-2 \epsilon)^{2}}
$$

and the response of the two feedback systems $[\mathbf{G}, \mathbf{H}]$ and $\left[\mathbf{G}_{1}, \mathbf{H}\right]$ with zero external excitation signals satisfy

$$
\left\|\sigma \boldsymbol{\Pi}_{\mathcal{M} \| \mathcal{N}} 0-\Pi_{\mathcal{M}_{1} \| \mathcal{N}} 0\right\|_{\infty} \leq \frac{2 \epsilon}{1-\epsilon} .
$$

Equation (10) shows that the trajectories of the nominal and perturbed systems are close in a peak sense, when one of the two is scaled appropriately in time. This shows that oscillations persist in the perturbed system, albeit with different and possibly varying periods. The proof of the theorem will be done in two steps. First, we consider the response of the autonomous system $[\mathbf{G}, \mathbf{H}]$ (i.e., with $w_{0}=0$ ), and we obtain bounds on the perturbation of the response when a small nonzero disturbance signal $x_{0} \neq 0$ is applied. This step is intended to bound the term

$$
\sigma \Pi_{\mathcal{M} \| \mathcal{N}} w_{0}-\Pi_{\mathcal{M} \| \mathcal{N}} x_{0}
$$

in the right hand side of equation (8) when $x_{0}$ is close to $w_{0}=0$. Second, we bring in the perturbed system $\mathbf{G}_{1}$ and analyze equation (7) to establish a bound on the signal $x_{0}$. This is achieved by considering a modified relay-hysteresis as an intermediate step. This part of the proof is intended to bound the term

$$
\left(\mathbf{I}-\Phi_{\mathcal{M}}\right) \boldsymbol{\Pi}_{\mathcal{M} \| \mathcal{N}} x_{0}
$$

in equation (8).

\section{A. Effect of disturbances on nominal trajectory}

We first consider the nominal trajectory of the unforced oscillator $[\mathbf{G}, \mathbf{H}]$ and compare it with the response to a small disturbance signal.

\section{A.1 Nominal trajectory of $[\mathbf{G}, \mathbf{H}]$}

The unforced response (i.e., with $w_{0}=0$ ) of the nominal system $[\mathbf{G}, \mathbf{H}]$ is shown in Figure 3 . The shape of the response is a direct consequence of the fact that the negative integrator $\mathbf{G}$ is driven by $u_{1}=-u_{2}= \pm 1$, and this value remains constant until the input $y_{2}=-y_{1}$ to the relay reaches the value \pm 1 , at which point the relay switches and $u_{1}$ changes sign. The switching times are $t_{1}=1$, and $t_{k}=t_{k-1}+2$ for $k>1$. 

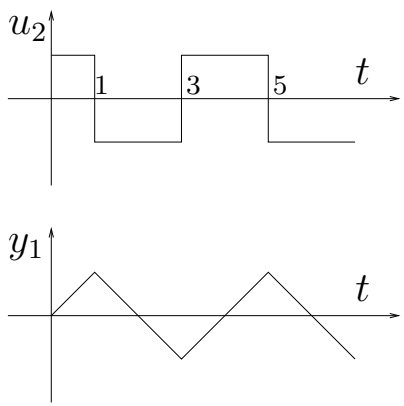

Fig. 3. Autonomous response of relay oscillator

\section{A.2 Bounds on the forced response and time-scaling function}

We now consider a disturbance $w_{0}=x_{0} \neq 0$ applied to the nominal system $[\mathbf{G}, \mathbf{H}]$ where $\left\|x_{0}\right\|_{\infty}=$ $r<1$. We show that the response retains the oscillatory nature, and we construct an appropriate scaling function $\sigma$ so that $\sigma \Pi_{\mathcal{M} \| \mathcal{N}} 0$ is close to $\Pi_{\mathcal{M} \| \mathcal{N}} x_{0}$ in the sense of Section III.

We denote the signals in the loop by $u_{1}, y_{1}$ etc. when the external disturbance is $w_{0}=0$, and by $u_{1}^{\prime}, y_{1}^{\prime}$ etc. when the external disturbance is $w_{0}=x_{0}=\left(\begin{array}{c}u_{0}^{\prime} \\ y_{0}^{\prime}\end{array}\right)$ with $\left\|u_{0}^{\prime}\right\|_{\infty},\left\|y_{0}^{\prime}\right\|_{\infty} \leq r$. Similarly we denote the switching times by $t_{1}=1, t_{2}=3, \ldots$ and $t_{1}^{\prime}, t_{2}^{\prime}, \ldots$ in the two cases, respectively. We first claim that the sequence $t_{1}^{\prime}, t_{2}^{\prime}, \ldots$ is infinite. To see this observe that, for small $t$,

$$
y_{1}^{\prime}(t)=\int_{0}^{t}\left(1-u_{0}^{\prime}(\tau)\right) d \tau,
$$

which is strictly increasing. Moreover $y_{2}^{\prime}(t)=y_{0}^{\prime}(t)-y_{1}^{\prime}(t) \leq r-(1-r) t$, so $y_{2}^{\prime}(t)$ eventually reaches -1 at which time the first switch occurs. Thereafter $y_{1}^{\prime}(t)$ becomes strictly decreasing and there is a further switch, etc. We also point out that there can be no finite limit point of switching times (Proposition 2).

We now define a suitable $\mathcal{K}_{\infty}$-function $\sigma$ which maps the sequence $t_{1}^{\prime}, t_{2}^{\prime}, \ldots$ onto $t_{1}=1, t_{2}=3, \ldots$ and leads to $\sigma \Pi_{\mathcal{M} \| \mathcal{N}} 0-\Pi_{\mathcal{M} \| \mathcal{N}} x_{0}$ which is small in norm. On the interval $\left[t_{k}^{\prime}, t_{k+1}^{\prime}\right]$ we define

$$
\sigma(t):=t_{k}+2 \frac{y_{1}^{\prime}(t)-y_{1}^{\prime}\left(t_{k}^{\prime}\right)}{y_{1}^{\prime}\left(t_{k+1}^{\prime}\right)-y_{1}^{\prime}\left(t_{k}^{\prime}\right)}
$$

while on $\left[0, t_{1}^{\prime}\right]$,

$$
\sigma(t):=\frac{y_{1}^{\prime}(t)-y_{1}^{\prime}(0)}{y_{1}^{\prime}\left(t_{1}^{\prime}\right)-y_{1}^{\prime}(0)}=\frac{y_{1}^{\prime}(t)}{y_{1}^{\prime}\left(t_{1}^{\prime}\right)} .
$$

Observe that $\sigma\left(t_{k}^{\prime}\right)=t_{k}$ for all $k$, and that $\sigma(\cdot)$ is monotonically increasing. Now observe that on the interval $\left[t_{k}^{\prime}, t_{k+1}^{\prime}\right]$ for $k \geq 1$ :

$$
\sigma y_{1}(t)-y_{1}^{\prime}(t)=(-1)^{k}\left(-1+\sigma(t)-t_{k}\right)-y_{1}^{\prime}(t),
$$

while on the interval $\left[0, t_{1}^{\prime}\right]$ since $y_{1}(t)=t$ :

$$
\sigma y_{1}(t)-y_{1}^{\prime}(t)=\sigma(t)-y_{1}^{\prime}(t) .
$$

In both cases, $\sigma y_{1}(t)-y_{1}^{\prime}(t)$ is a linear function of $y_{1}^{\prime}(t)$. Hence it is monotonic on each such interval. But

$$
\begin{aligned}
\left|\sigma y_{1}\left(t_{k}^{\prime}\right)-y_{1}^{\prime}\left(t_{k}^{\prime}\right)\right| & =\left|(-1)^{k+1}-y_{1}^{\prime}\left(t_{k}^{\prime}\right)\right| \\
& =\left|(-1)^{k+1}-\left(y_{0}^{\prime}\left(t_{k}^{\prime}\right)-y_{2}^{\prime}\left(t_{k}^{\prime}\right)\right)\right| \\
& =\left|-y_{0}^{\prime}\left(t_{k}^{\prime}\right)\right| \leq r .
\end{aligned}
$$


This holds for all $k \geq 1$ while for $t=0$ we have that $\left|\sigma y_{1}(0)-y_{1}^{\prime}(0)\right|=0$. Together with monotonicity this shows that

$$
\left|\sigma y_{1}(t)-y_{1}^{\prime}(t)\right| \leq r
$$

for all values of $t \in[0, \infty)$. Since $\sigma u_{2}=u_{2}^{\prime}$ while $u_{0}=0$ and $\left\|u_{0}^{\prime}\right\|_{\infty} \leq r$, then

$$
\left\|\sigma u_{1}(t)-u_{1}^{\prime}(t)\right\|_{\infty} \leq r .
$$

We conclude that

$$
\left\|\sigma \Pi_{\mathcal{M} \| \mathcal{N}} 0-\Pi_{\mathcal{M} \| \mathcal{N}} x_{0}\right\|_{\infty} \leq r=\left\|x_{0}\right\|_{\infty} .
$$

Next we establish a bound on the amount of "time stretching" which $\sigma$ introduces. On the interval $\left[t_{k}^{\prime}, t_{k+1}^{\prime}\right]$ we have

$$
\begin{aligned}
y_{1}^{\prime}(t) & =y_{1}^{\prime}\left(t_{k}^{\prime}\right)-\int_{t_{k}^{\prime}}^{t} u_{1}^{\prime}(\tau) d \tau \\
& =y_{1}^{\prime}\left(t_{k}^{\prime}\right)-\int_{t_{k}^{\prime}}^{t}\left(u_{0}^{\prime}(\tau)+\operatorname{sgn}\left(y_{1}^{\prime}\left(t_{k}^{\prime}\right)\right)\right) d \tau \\
& =y_{1}^{\prime}\left(t_{k}^{\prime}\right)-\left(t-t_{k}^{\prime}\right) \operatorname{sgn}\left(y_{1}^{\prime}\left(t_{k}^{\prime}\right)\right)-\int_{t_{k}^{\prime}}^{t} u_{0}^{\prime}(\tau) d \tau .
\end{aligned}
$$

Using the bound $\left\|u_{0}^{\prime}\right\|_{\infty} \leq r$ we obtain:

$$
\left|y_{1}^{\prime}\left(t_{k+1}^{\prime}\right)-y_{1}^{\prime}\left(t_{k}^{\prime}\right)+\left(t_{k+1}^{\prime}-t_{k}^{\prime}\right) \operatorname{sgn}\left(y_{1}^{\prime}\left(t_{k}^{\prime}\right)\right)\right| \leq\left(t_{k+1}^{\prime}-t_{k}^{\prime}\right) r
$$

which gives the bounds:

$$
\left(t_{k+1}^{\prime}-t_{k}^{\prime}\right)(1-r) \leq\left|y_{1}^{\prime}\left(t_{k+1}^{\prime}\right)-y_{1}^{\prime}\left(t_{k}^{\prime}\right)\right| \leq\left(t_{k+1}^{\prime}-t_{k}^{\prime}\right)(1+r) .
$$

From $y_{2}^{\prime}=y_{0}^{\prime}-y_{1}^{\prime}$, the bound $\left\|y_{0}^{\prime}\right\|_{\infty} \leq r$ and the fact that $y_{2}^{\prime}(t)= \pm 1$ at successive switching times we also have the bounds

$$
2(1-r) \leq\left|y_{1}^{\prime}\left(t_{k+1}^{\prime}\right)-y_{1}^{\prime}\left(t_{k}^{\prime}\right)\right| \leq 2(1+r) .
$$

Combining (16) and (17) we deduce that

$$
2 \frac{1-r}{1+r} \leq t_{k+1}^{\prime}-t_{k}^{\prime} \leq 2 \frac{1+r}{1-r}
$$

Since $y_{1}^{\prime}(0)=0$, the factor 2 can be removed in (17) when $k=0$ so that we obtain the bounds

$$
\frac{1-r}{1+r} \leq t_{1}^{\prime} \leq \frac{1+r}{1-r}
$$

Summing up (18) for successive values of $k$, adding (19) and using the fact that $t_{k}=2 k-1$ we obtain that

$$
t_{k} \frac{1-r}{1+r} \leq t_{k}^{\prime} \leq t_{k} \frac{1+r}{1-r}
$$

and then, that

$$
\left|t_{k}^{\prime}-t_{k}\right| \leq \frac{2 r}{1-r} t_{k} \leq \frac{2 r}{1-r} \frac{1+r}{1-r} t_{k}^{\prime}
$$

Returning to (15) and substituting into (12) we obtain that for $t \in\left[t_{k}^{\prime}, t_{k+1}^{\prime}\right]$,

$$
\sigma(t)-t=\alpha\left(t-t_{k}^{\prime}\right)-\left(t-t_{k}\right)-2 \frac{\int_{t_{k}^{\prime}}^{t} u_{0}^{\prime}(\tau) d \tau}{y_{1}^{\prime}\left(t_{k+1}^{\prime}\right)-y_{1}^{\prime}\left(t_{k}^{\prime}\right)}
$$


where

$$
\alpha:=-2 \frac{\operatorname{sgn}\left(y_{1}^{\prime}\left(t_{k}^{\prime}\right)\right)}{y_{1}^{\prime}\left(t_{k+1}^{\prime}\right)-y_{1}^{\prime}\left(t_{k}^{\prime}\right)} .
$$

It follows from (17) that $\frac{1}{1+r} \leq \alpha \leq \frac{1}{1-r}$ and then, that $|\alpha-1| \leq \frac{r}{1-r}$. Thus from (20), for $t \in\left[t_{k}^{\prime}, t_{k+1}^{\prime}\right]$

$$
\begin{aligned}
|\sigma(t)-t| & \leq|\alpha-1| t+\left|\alpha t_{k}^{\prime}-t_{k}\right|+2 \frac{r\left(t-t_{k}^{\prime}\right)}{2(1-r)} \\
& \leq|\alpha-1| t+\left|\alpha t_{k}^{\prime}-t_{k}^{\prime}\right|+\left|t_{k}^{\prime}-t_{k}\right|+\frac{r\left(t-t_{k}^{\prime}\right)}{(1-r)} \\
& \leq 2|\alpha-1| t+\left|t_{k}^{\prime}-t_{k}\right|+\frac{r\left(t-t_{k}^{\prime}\right)}{(1-r)} \\
& \leq 2 \frac{r}{1-r} t+\frac{2 r}{1-r} \frac{1+r}{1-r} t_{k}^{\prime}+\frac{r}{1-r}\left(t-t_{k}^{\prime}\right) \\
& \leq 3 \frac{r}{1-r} t+\left(\frac{2 r}{1-r} \frac{1+r}{1-r}-\frac{r}{1-r}\right) t_{k}^{\prime} \\
& \leq 4 \frac{r}{(1-r)^{2}} t .
\end{aligned}
$$

Hence

$$
\left|\frac{\sigma(t)-t}{t}\right| \leq \frac{4 r}{(1-r)^{2}}
$$

We now turn to the interval $\left[0, t_{1}^{\prime}\right]$. Substituting (11) into (13) and rearranging terms gives

$$
\sigma(t)-t=\frac{t-\int_{0}^{t} u_{0}^{\prime}(\tau) d \tau}{y_{1}^{\prime}\left(t_{1}^{\prime}\right)}-t
$$

whence

$$
\begin{aligned}
|\sigma(t)-t| & \leq t\left|\frac{y_{1}^{\prime}\left(t_{1}^{\prime}\right)-1}{y_{1}^{\prime}\left(t_{1}^{\prime}\right)}\right|+t \frac{r}{\left|y_{1}^{\prime}\left(t_{1}^{\prime}\right)\right|} \\
& \leq t \frac{r}{1-r}+t \frac{r}{1-r}=2 t \frac{r}{1-r},
\end{aligned}
$$

which is tighter than the bound in (21). Thus (21) holds for all $t$.

\section{$B$. The effect of modelling uncertainty}

We now continue with the proof of Theorem 1 which is built around the key equations (7) and (8). Accordingly we now consider a perturbed system $\mathbf{G}_{1}$ with graph $\mathcal{M}_{1}$ for which there is a bijective mapping $\boldsymbol{\Phi}_{\mathcal{M}}: \mathcal{M} \rightarrow \mathcal{M}_{1}$.

\section{B.1 Modified relay-hysteresis}

In order to proceed with the proof, a key step is to bound the norm of $x_{0}$ which solves equation (7). For this, a difficulty arises from the fact that the response of the nominal system $[\mathbf{G}, \mathbf{H}]$ is not globally bounded. Indeed, a constant input signal $u_{0}$ of amplitude $>1$ will result in a non-oscillatory behaviour where the output $y_{1}$ of the integrator $\mathbf{G}$ ramps up to $\infty$. Thus, while we easily obtain that

$$
\left\|x_{0}\right\|_{\infty}=\left\|\left(\Phi_{\mathcal{M}}-\mathbf{I}\right) \boldsymbol{\Pi}_{\mathcal{M} \| \mathcal{N}} x_{0}\right\|_{\infty}
$$

from (7), the unboundedness of $\boldsymbol{\Pi}_{\mathcal{M} \| \mathcal{N}}$ prevents a bound on $\left\|x_{0}\right\|_{\infty}$ from being deduced directly. 
The approach in $[10$, Section IV] which makes use of degree theory for analyzing the solution of the feedback equation on bounded sets appears quite challenging in the present context. Hence, we follow an alternative route. We consider a modified system, shown in Figure 4, where the relay has been replaced by $\mathbf{H}_{0}$ whose output increases linearly with slope $\alpha$ in the region $\left|y_{2}\right| \geq c>1$. This new system will be shown next to be globally bounded. Yet, for small enough inputs, the responses of both $[\mathbf{G}, \mathbf{H}]$ and $\left[\mathbf{G}, \mathbf{H}_{0}\right]$ are identical. This will eventually allow us to obtain the required bounds of Theorem 1 for the unperturbed hysteresis $\mathbf{H}$. Following our earlier convention we denote the graph of $\mathbf{H}_{0}$ by $\mathcal{N}_{0}$.

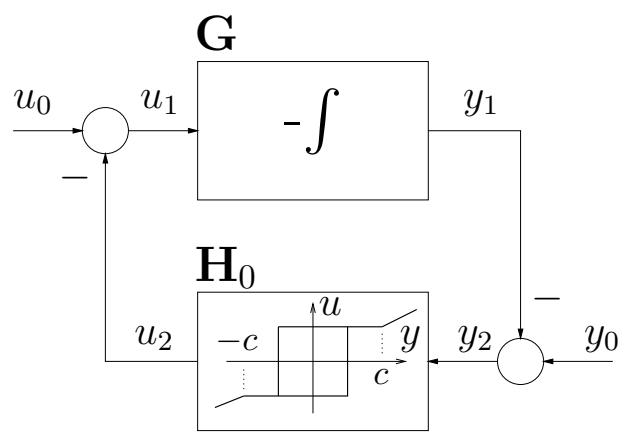

Fig. 4. Globally bounded relay oscillator

Proposition 4: Let $\left\|x_{0}\right\|_{\infty}=r$. Then $\left\|\boldsymbol{\Pi}_{\mathcal{M} \| \mathcal{N}_{0}} x_{0}\right\|_{\infty} \leq f(r)$ where

$$
f(r)= \begin{cases}1+r+\alpha \max \{0,1+2 r-c\}, & \text { when } r<1, \text { and } \\ \max \left\{c-\alpha^{-1}+\left(1+\alpha^{-1}\right) r, 2(1+\alpha) r\right\}, & \text { when } r \geq 1 .\end{cases}
$$

Proof: We introduce the notation $h_{0}(x)$ for a multi-valued function which contains the range values of $\mathbf{H}_{0}$ at any point in time, i.e. $\mathbf{H}_{0}(x)(t) \in h_{0}(x(t))$. This is given by

$$
h_{0}(x)= \begin{cases}\{+1,-1\} & \text { when }|x| \leq 1 \\ \mathbf{H}_{0}(x) & \text { when }|x|>1\end{cases}
$$

The solutions of the feedback system of Figure 4 are determined by the integral equation

$$
y_{1}(t)=\int_{0}^{t}\left(\mathbf{H}_{0}\left(y_{0}-y_{1}\right)(\tau)-u_{0}(\tau)\right) d \tau .
$$

It follows that if $M_{0}>0$ is such that $h_{0}\left(y_{0}(\tau)-M\right)-u_{0}(\tau) \leq 0$ for all $\tau \in[0, t]$ and all $M \geq M_{0}$, then $y_{1}(t) \leq M_{0}$. (To see this, assume to the contrary, and consider an interval $\left[t_{1}, t\right]$ where $y\left(t_{1}\right)=M_{0}$, $y(\tau) \geq M_{0}$ for $\tau \in\left[t_{1}, t\right]$, and $y(t)>M_{0}$. Since the integrand is $\leq 0$ in the interval $\left[t_{1}, t\right]$ it follows that $y(t) \leq y\left(t_{1}\right)$ which is a contradiction.)

We consider solutions under the condition that $\left\|u_{0}\right\|_{\infty},\left\|y_{0}\right\|_{\infty} \leq r$. It follows that if

$$
h_{0}\left(y_{0}-M\right)-u_{0} \leq 0
$$

for all $M \geq M_{0}$ and all values $\left|u_{0}\right|,\left|y_{0}\right| \leq r$, then $M_{0}>0$ is an upper bound for $\left\|y_{1}\right\|_{\infty}$. Condition (23) is equivalent to

$$
h_{0}\left(r-M_{0}\right) \leq-r .
$$

If $r<1$ this holds providing $M_{0}>1+r$. If $r \geq 1$, it holds providing $r-M_{0} \leq \xi$ where $\xi$ satisfies $-1+\alpha(\xi+c)=-r$, which then gives a bound $M_{0} \geq r+c+\alpha^{-1}(r-1)$. We therefore deduce the bounds:

$$
\left\|y_{1}\right\|_{\infty} \leq \begin{cases}1+r & \text { when } r<1 \\ c-\alpha^{-1}+\left(1+\alpha^{-1}\right) r & \text { when } r \geq 1\end{cases}
$$


Under the same conditions $\left\|u_{0}\right\|_{\infty},\left\|y_{0}\right\|_{\infty} \leq r$, since $\left|y_{2}\right| \leq r+\left|y_{1}\right|,\left|u_{2}\right| \leq \max \left\{1,1+\alpha\left(\left|y_{2}\right|-c\right)\right\}$, $\left|u_{1}\right| \leq\left|u_{2}\right|+r$ we deduce the following bounds for $u_{1}$ :

$$
\left\|u_{1}\right\|_{\infty} \leq \begin{cases}1+r+\alpha \max \{0,1+2 r-c\} & \text { when } r<1 \\ 2(1+\alpha) r & \text { when } r \geq 1\end{cases}
$$

Remark. For the analysis of the next section, a favourable choice of the parameters $c$ and $\alpha$ is $c=2$ and $\alpha=1 / 2$, which gives:

$$
f(r)= \begin{cases}1+r & \text { when } r \leq \frac{1}{2} \\ \frac{1}{2}+2 r & \text { when } \frac{1}{2}<r<1 \\ 3 r & \text { when } 1 \leq r .\end{cases}
$$

This choice of parameters gives the least conservative upper bound for $\epsilon$ in Theorem 1 for this manner of proof.

\section{B.2 Derivation of bounds for the perturbed system and proof of Theorem 1}

Our next step is to consider the fundamental equations (7) and (8) but with $\mathbf{H}$ replaced by $\mathbf{H}_{0}$ and $c=2, \alpha=1 / 2$ in the definition of $\mathbf{H}_{0}$. Namely we consider the equation:

$$
\left(\sigma \boldsymbol{\Pi}_{\mathcal{M} \| \mathcal{N}_{0}}-\boldsymbol{\Pi}_{\mathcal{M}_{1} \| \mathcal{N}_{0}}\right) 0=\left(\mathbf{I}-\boldsymbol{\Phi}_{\mathcal{M}}\right) \boldsymbol{\Pi}_{\mathcal{M} \| \mathcal{N}_{0}} x_{0}+\sigma \boldsymbol{\Pi}_{\mathcal{M} \| \mathcal{N}_{0}} 0-\boldsymbol{\Pi}_{\mathcal{M} \| \mathcal{N}_{0}} x_{0}
$$

for some $\sigma \in \mathcal{K}_{\infty}$, where

$$
0=\left(\mathbf{I}+\left(\Phi_{\mathcal{M}}-\mathbf{I}\right) \boldsymbol{\Pi}_{\mathcal{M} \| \mathcal{N}_{0}}\right) x_{0} .
$$

We point out that (26) and (27) hold providing that $\left[\mathbf{G}, \mathbf{H}_{0}\right]$ and $\left[\mathbf{G}_{1}, \mathbf{H}_{0}\right]$ are well-posed feedback systems. The proofs of these facts are similar to the proofs of Propositions 2 and 3 and are omitted. Let $\left\|\Phi_{\mathcal{M}}-\mathbf{I}\right\| \leq \epsilon$. Then from (27) and Proposition 4 we obtain

$$
\begin{aligned}
\left\|x_{0}\right\|_{\infty} & =\left\|\left(\boldsymbol{\Phi}_{\mathcal{M}}-\mathbf{I}\right) \boldsymbol{\Pi}_{\mathcal{M} \| \mathcal{N}_{0}} x_{0}\right\|_{\infty} \\
& \leq \epsilon f\left(\left\|x_{0}\right\|_{\infty}\right) .
\end{aligned}
$$

In order to obtain an upper bound for $\left\|x_{0}\right\|_{\infty}$ from (29) it is necessary that $\epsilon<1 / 3$, since otherwise the inequality is satisfied whenever $\left\|x_{0}\right\|_{\infty} \geq 1$ from (25). If $\epsilon<1 / 3$ we observe that (29) fails whenever $\left\|x_{0}\right\|_{\infty} \geq 1 / 2$, from (25). Thus, if $\epsilon<1 / 3$ we conclude that $\left\|x_{0}\right\|_{\infty}<1 / 2$, in which case (29) is equivalent to $\left\|x_{0}\right\|_{\infty} \leq \epsilon\left(1+\left\|x_{0}\right\|_{\infty}\right)$. This gives the upper bound:

$$
\left\|x_{0}\right\|_{\infty} \leq \frac{\epsilon}{1-\epsilon}<\frac{1}{2}
$$

Under the same condition that $\epsilon<1 / 3$, from (25) and (30) we have the bound:

$$
\left\|\boldsymbol{\Pi}_{\mathcal{M} \| \mathcal{N}_{0}} x_{0}\right\|_{\infty} \leq 1+\frac{\epsilon}{1-\epsilon}=\frac{1}{1-\epsilon}
$$

Since $\boldsymbol{\Pi}_{\mathcal{M} \| \mathcal{N}_{0}}+\boldsymbol{\Pi}_{\mathcal{N}_{0} \| \mathcal{M}}=\mathbf{I}$, this gives:

$$
\begin{aligned}
\left\|\boldsymbol{\Pi}_{\mathcal{N}_{0} \| \mathcal{M}} x_{0}\right\|_{\infty} & \leq\left\|x_{0}\right\|_{\infty}+\left\|\boldsymbol{\Pi}_{\mathcal{M} \| \mathcal{N}_{0}} x_{0}\right\|_{\infty} \\
& \leq \frac{\epsilon}{1-\epsilon}+\frac{1}{1-\epsilon}=\frac{1+\epsilon}{1-\epsilon}
\end{aligned}
$$

We deduce that, providing

$$
\frac{1+\epsilon}{1-\epsilon} \leq c=2
$$


which is equivalent to $\epsilon \leq 1 / 3$, the system $\left[\mathbf{G}, \mathbf{H}_{0}\right]$ never produces an input to $\mathbf{H}_{0}$ which exceeds $c$, which then means that

$$
\Pi_{\mathcal{M} \| \mathcal{N}_{0}} x_{0}=\Pi_{\mathcal{M} \| \mathcal{N}} x_{0}
$$

i.e. $\left[\mathbf{G}, \mathbf{H}_{0}\right]$ and $[\mathbf{G}, \mathbf{H}]$ have the same response to the disturbance $x_{0}$. Now observe that

$$
\Pi_{\mathcal{M} \| \mathcal{N}_{0}} 0=\Pi_{\mathcal{M} \| \mathcal{N}} 0
$$

since $\left|y_{1}(t)\right|$ never exceeds one in the response of $[\mathbf{G}, \mathbf{H}]$ to a disturbance $w_{0}=0$ (see Section IV-A.1), and the same is then true for $\left[\mathbf{G}, \mathbf{H}_{0}\right]$. We therefore conclude, using (32), (33), and (14), that there exists a $\sigma \in \mathcal{K}_{\infty}$ such that

$$
\begin{aligned}
\left\|\sigma \Pi_{\mathcal{M} \| \mathcal{N}_{0}} 0-\Pi_{\mathcal{M} \| \mathcal{N}_{0}} x_{0}\right\|_{\infty} & =\left\|\sigma \Pi_{\mathcal{M} \| \mathcal{N}} 0-\Pi_{\mathcal{M} \| \mathcal{N}} x_{0}\right\|_{\infty} \\
& \leq\left\|x_{0}\right\|_{\infty} \leq \frac{\epsilon}{1-\epsilon}
\end{aligned}
$$

providing $\epsilon<1 / 3$. Moreover, from (21), we have the bound

$$
\left|\frac{\sigma(t)-t}{t}\right| \leq \frac{4\left\|x_{0}\right\|_{\infty}}{\left(1-\left\|x_{0}\right\|_{\infty}\right)^{2}} \leq \frac{4 \epsilon(1-\epsilon)}{(1-2 \epsilon)^{2}}
$$

We now return to (26) to obtain using (28) and (34) the bound:

$$
\left\|\left(\sigma \boldsymbol{\Pi}_{\mathcal{M} \| \mathcal{N}_{0}}-\Pi_{\mathcal{M}_{1} \| \mathcal{N}_{0}}\right) 0\right\|_{\infty} \leq 2\left\|x_{0}\right\|_{\infty} \leq \frac{2 \epsilon}{1-\epsilon}
$$

providing $\epsilon<1 / 3$. Equation (36) implies that

$$
\begin{aligned}
\left\|\boldsymbol{\Pi}_{\mathcal{N}_{0} \| \mathcal{M}_{1}} 0\right\|_{\infty} & =\left\|\boldsymbol{\Pi}_{\mathcal{M}_{1} \| \mathcal{N}_{0}} 0\right\|_{\infty} \\
& \leq\left\|\sigma \boldsymbol{\Pi}_{\mathcal{M} \| \mathcal{N}_{0}} 0\right\|_{\infty}+2\left\|x_{0}\right\|_{\infty} \\
& \leq 1+\frac{2 \epsilon}{1-\epsilon}
\end{aligned}
$$

Thus, if

$$
1+\frac{2 \epsilon}{1-\epsilon} \leq c=2,
$$

which is equivalent to $\epsilon \leq 1 / 3$, the system $\left[\mathbf{G}_{1}, \mathbf{H}_{0}\right]$ never produces an input to $\mathbf{H}_{0}$ which exceeds $c$, which then means that

$$
\Pi_{\mathcal{M}_{1} \| \mathcal{N}_{0}} 0=\Pi_{\mathcal{M}_{1} \| \mathcal{N}} 0
$$

and (36) then produces the bound

$$
\left\|\left(\sigma \boldsymbol{\Pi}_{\mathcal{M} \| \mathcal{N}}-\Pi_{\mathcal{M}_{1} \| \mathcal{N}}\right) 0\right\|_{\infty} \leq \frac{2 \epsilon}{1-\epsilon}
$$

Equation (38) is the same as equation (10), and equation (35) is the same as (9). These are established under the assumption that $\epsilon<1 / 3$. This completes the proof of Theorem 1 . 


\section{EXAmple}

We study the behaviour of the relaxation oscillator of Figure 2 when the nominal integrator, i.e. with transfer function $P(s)=-1 / s$, is replaced by a system with transfer function

$$
P^{\prime}(s)=\frac{-a e^{-h s}}{s+b}
$$

where $a>0$. We first study the case of $h=0$ since this is amenable to explicit calculation. We denote the solutions of the perturbed system by $u_{1}^{\prime}, y_{1}^{\prime}$, etc. Before the first hysteresis switch, the system evolves according to: $\dot{y}_{1}^{\prime}+b y_{1}^{\prime}=a$, which has solution

$$
y_{1}^{\prime}(t)=\left(1-e^{-b t}\right) \frac{a}{b},
$$

providing $b \neq 0$. If $b \geq a$, then the hysteresis never switches and the relaxation oscillation breaks down. So let us assume that $b<a$. After the switch the system evolves according to: $\dot{y}_{1}^{\prime}+b y_{1}^{\prime}=-a$. In order that $\dot{y}_{1}^{\prime}$ becomes negative just after the switch we require that $b>-a$, otherwise $\dot{y}_{1}^{\prime}(t)$ remains equal to one or escapes to $+\infty$, and again the relaxation oscillation breaks down. So let us assume that $|b|<a$. Under such a condition the hysteresis continues to switch at times $0<t_{1}^{\prime}<t_{2}^{\prime}<\ldots$, and in each interval $\left[t_{k}^{\prime}, t_{k+1}^{\prime}\right]$ the solution is:

$$
y_{1}^{\prime}(t)=(-1)^{k}\left(\frac{a}{b}-\left(1+\frac{a}{b}\right) e^{-b\left(t-t_{k}^{\prime}\right)}\right) .
$$

We now define a suitable scaling function $\sigma(t)$. On the interval $\left[t_{k}^{\prime}, t_{k+1}^{\prime}\right]$ we define

$$
\sigma(t)=t_{k}+(-1)^{k}\left(y_{1}^{\prime}(t)-y_{1}^{\prime}\left(t_{k}^{\prime}\right)\right)
$$

while on $\left[0, t_{1}^{\prime}\right], \sigma(t)=y_{1}^{\prime}(t)$. As before, observe that $\sigma$ is monotonically increasing, $\sigma\left(t_{k}^{\prime}\right)=t_{k}$ for all $k$ and $\sigma u_{1}(t)-u^{\prime}(t)=0$. Furthermore, on $\left[t_{k}^{\prime}, t_{k+1}^{\prime}\right]$ :

$$
\sigma y_{1}(t)-y_{1}^{\prime}(t)=(-1)^{k}\left(-1+\sigma(t)-t_{k}\right)-y_{1}^{\prime}(t)=0
$$

since $y_{1}^{\prime}\left(t_{k}^{\prime}\right)=(-1)^{k+1}$. The same fact holds on $\left[0, t_{1}^{\prime}\right]$, so we have shown that

$$
\sigma \Pi_{\mathcal{M} \| \mathcal{N}} 0=\Pi_{\mathcal{M}_{1} \| \mathcal{N}} 0 .
$$

The switching times are given by

$$
\begin{aligned}
t_{1}^{\prime} & =\frac{1}{b} \ln \frac{a}{a-b}, \\
t_{k+1}^{\prime} & =t_{k}^{\prime}+\frac{1}{b} \ln \frac{a+b}{a-b},
\end{aligned}
$$

and we easily check that $t_{k}^{\prime} \rightarrow t_{k}$ as $a \rightarrow 1$ and $b \rightarrow 0$. It can also be shown that

$$
\sup _{t} \frac{|\sigma(t)-t|}{t} \rightarrow 0
$$

as $a \rightarrow 1$ and $b \rightarrow 0$.

We now turn to the question of the "gap" between $\mathbf{G}$ and $\mathbf{G}^{\prime}$. We find that

$$
\begin{aligned}
\mathcal{M} & =\left[\begin{array}{c}
M \\
N
\end{array}\right] \mathcal{L}_{\infty}=\left[\begin{array}{c}
\frac{s}{s+1} \\
\frac{-1}{s+1}
\end{array}\right] \mathcal{L}_{\infty}, \\
\mathcal{M}^{\prime} & =\left[\begin{array}{c}
M_{1} \\
N_{1}
\end{array}\right] \mathcal{L}_{\infty}=\left[\begin{array}{c}
\frac{s+b}{s+1} \\
\frac{-a e^{-h s}}{s+1}
\end{array}\right] \mathcal{L}_{\infty},
\end{aligned}
$$


where, as customary, we represent by $G(s) \mathcal{L}_{\infty}$ the image of $\mathcal{L}_{\infty}[0, \infty)$ under the action of a convolution operator whose kernel is the inverse Laplace transforms of $G(s)$. Define $(V, U)=(1,-1)$ and note that $(V, U)\left(\begin{array}{c}M \\ N\end{array}\right)=1$. Thus,

$$
\boldsymbol{\Phi}:=\left(\begin{array}{c}
M_{1} \\
N_{1}
\end{array}\right)\left(\begin{array}{ll}
V & U
\end{array}\right)
$$

maps $\mathcal{M}$ onto $\mathcal{M}_{1}$ and we find that

$$
\begin{aligned}
\left.(\mathbf{I}-\boldsymbol{\Phi})\right|_{\mathcal{M}}= & \left(\begin{array}{c}
M \\
N
\end{array}\right)\left(\begin{array}{ll}
V & U
\end{array}\right) \\
& -\left(\begin{array}{c}
M_{1} \\
N_{1}
\end{array}\right)\left(\begin{array}{ll}
V & U
\end{array}\right) \\
= & \left(\begin{array}{c}
\frac{-b}{s+1} \\
\frac{a e^{-h s}-1}{s+1}
\end{array}\right)\left(\begin{array}{ll}
1 & -1
\end{array}\right) .
\end{aligned}
$$

It follows that

$$
\left\|\left.(\mathbf{I}-\mathbf{\Phi})\right|_{\mathcal{M}}\right\| \leq 2 \max \left\{\left\|\frac{b}{s+1}\right\|,\left\|\frac{1-a e^{-h s}}{s+1}\right\|\right\}
$$

where $\|\cdot\|$ in the righthand side of the above equation denotes the induced norm of the relevant operators in an $\mathcal{L}_{\infty}$ sense, i.e.,

$$
\begin{aligned}
\left\|\frac{b}{s+1}\right\| & =\left\|b e^{-t}\right\|_{\mathcal{L}_{1}}=|b|, \text { while } \\
\left\|\frac{1-a e^{-h s}}{s+1}\right\| & =\int_{0}^{h} e^{-t} d t+\int_{h}^{\infty}\left|e^{-h}-a\right| e^{h-t} d t=1-e^{-h}+\left|e^{-h}-a\right|,
\end{aligned}
$$

being in both cases the $\mathcal{L}_{1}$ norm of the respective convolution kernels. Hence, Theorem 1 predicts that oscillations will not break down providing

$$
\max \left\{|b|, 1-e^{-h}+\left|e^{-h}-a\right|\right\}<\frac{1}{6} .
$$

In contrast, by direct calculation we were able to show for the case $h=0$, that oscillations do not break down if $|b|<a$, which is consistent with condition (39).

\section{Summary}

Theorem 1 gives a sufficient condition for robustness of oscillatory behaviour of the relay relaxation oscillator. It states that, as long as a perturbation of the dynamical component is sufficiently small $(<1 / 3)$ in a gap sense, oscillations persist.

\section{REFERENCES}

[1] K. J. Astrom, T. H. Lee, K. K. Tan, and K. H. Johansson, "Recent advances in relay feedback methods-a survey," in Proceedings of the IEEE Conference on Systems, Man and Cybernetics, vol. 3, (Piscataway, NJ), pp. $2616-2621,1995$.

[2] A. R. Bergen and R. L. Franks, "Justification of the Describing Function Method," SIAM J. Control and Optimization, 9(4): 568-589, 1971.

[3] A. R. Bulsara and L. Gammaitoni, Tuning in to noise, Physics Today, March 1996, pp. 39-45.

[4] C.A. Desoer and M. Vidyasagar, Feedback systems: Input-output properties, Academic Press: New York, 1975.

[5] J.C. Doyle, T.T. Georgiou, and M.C. Smith, On the parallel projection of nonlinear systems, Systems 86 Control Letters, 20, 79-85, 1993.

[6] C. Foias, T.T. Georgiou, and M.C. Smith, Geometric techniques for robust stabilization of linear time-varying systems, Proc. of the 1990 IEEE Conf. on Decision 8 Control, pp. 2868-2873; Robust stability of feedback systems: A geometric approach using the gap metric, SIAM Journal on Control and Optimization, 31, 1518-37, 1993. 
[7] T.T. Georgiou, Differential stability and robust control of nonlinear systems, Mathematics of Control, Signals and Systems, 6, 289-307, 1993.

[8] T.T. Georgiou and M.C. Smith, Optimal robustness in the gap metric, IEEE Trans. on Automat. Control, 35, 673-686, 1990.

[9] T.T. Georgiou and M.C. Smith, Metric Uncertainty and Nonlinear Feedback Stabilization, in Feedback Control, Nonlinear Systems, and Complexity, B.A. Francis and A.R. Tannenbaum (Editors), Springer-Verlag, Lecture Notes in Control and Information Sciences, vol. 202, pp. 88-98, 1995.

[10] T.T. Georgiou and M.C. Smith, Robustness Analysis of Nonlinear Feedback Systems: An input-output approach, IEEE Trans. on Aut. Contr., 42(9): 1200-21, September 1997.

[11] T.T. Georgiou and M.C. Smith, Biased norms and metric uncertainty for nonlinear feedback systems, Proc. of the 1997 IEEE Conf. on Decision 83 Control, pp. 642-643.

[12] J. Grasman, Asymptotic Methods for Relaxation Oscillators and Applications, Springer-Verlag, 1987.

[13] J. Guckenheimer, "Dynamics of the Van der Pol equation," IEEE Trans. Circuits Syst., 27, pp. 983-989, November 1980.

[14] J. Hauser and D.G. Meyer, The trajectory manifold of a nonlinear control system, Proc. of the 1998 IEEE Conf. on Decision E Control, pp. 1034-9.

[15] E. A. Jackson, Perspectives of nonlinear dynamics, Cambridge University Press, 1991.

[16] U. Jönsson, C-Y Kao, and A. Megretski, Analysis of periodic orbits based on uncertain data, Proc. of the 1998 IEEE Conf. on Decision \& Control, pp. 4400-4405.

[17] A.N. Kolmogorov and S.V. Fomin, Introductory Real Analysis, Dover, 1975.

[18] R. Kippenhahn, 100 Billion Suns: The birth, life, and death of the stars, Princeton University Press, 1983.

[19] A. I. Mees and A. R. Bergen, "Describing functions revisited," IEEE Trans. on Autom. Control, AC 20(4): 473-478, 1975.

[20] A. Megretski, Global stability of oscillations induced by a relay feedback, IFAC, Budapest, Hungary, E:49-54, 1996.

[21] J.D. Murray, Mathematical Biology, Springer, 1993.

[22] W. Rudin, Real and Complex Analysis, 3nd edition, McGraw Hill, 1987.

[23] M. Safonov, Stability and Robustness of Multivariable Feedback System, MIT Press, 1980.

[24] Ya.Z. Tsypkin, Relay Control Systems, transl. C. Constanda, Cambridge University Press, 1984.

[25] S. Varigonda and T.T. Georgiou, Oscillations in systems with relay hysteresis, IEEE Trans. on Autom. Control, accepted for publication.

[26] M. Vidyasagar, H. Schneider and B. Francis, Algebraic and topological aspects of feedback stabilization, IEEE Trans. on Automat. Control, 27, 880-894, 1982.

[27] G. Vinnicombe, Frequency domain uncertainty and the graph topology, IEEE Trans. on Automat. Control, 38, 1371-1383, 1993.

[28] J. C. Willems, The Analysis of Feedback Systems, Cambridge, MA, M.I.T. Press, 1971.

[29] A.T. Winfree, When Time Breaks Down, Princeton University Press, 1986.

[30] G. Zames, Functional analysis applied to nonlinear feedback systems, IEEE Trans. on Circuit Theory, 10, 392-404, September 1963.

[31] G. Zames, Realizability conditions for nonlinear feedback systems, IEEE Trans. on Circuit Theory, 11, 186-194, June 1964.

[32] G. Zames, On the input-output stability of time-varying nonlinear feedback systems. Part I: Conditions using concepts of loop gain, conicity, and positivity, IEEE Trans. on Automat. Control, 11, 228-238, 1966.

[33] G. Zames, On the input-output stability of time-varying nonlinear feedback systems. Part II: Conditions involving circles in the frequency plane and sector nonlinearities, IEEE Trans. on Automat. Control, 11, 465-476, 1966.

[34] G. Zames and A.K. El-Sakkary, Unstable systems and feedback: The gap metric, Proceedings of the Allerton Conference, pp. 380-385, October 1980. 